\title{
Effect of grinding procedures on the ritonavir-lopinavir system
}

\section{B. M. Pinheiro', E. Culbertson², G. L. B. de Araujo ${ }^{3}$, S. Billinge ${ }^{2}$, F. F. Ferreira ${ }^{1,4}$}

${ }^{1}$ Federal University of ABC (UFABC), Santo André, SP, Brazil, ${ }^{2}$ Columbia University, New York, USA, ${ }^{3}$ University of São Paulo, São Paulo, SP, Brazil, " Nanomedicine Research Unit (NANOMED), Federal University of ABC (UFABC), Santo André, SP, Brazil

\author{
fabio.furlan@ufabc.edu.br
}

Ritonavir is a drug of the protease inhibitor class, marketed by Abbvie ${ }^{\mathbf{T M}}$ - as of 1996 under the name of Norvir ${ }^{\circledR}$ - for the treatment of adult and pediatric patients infected with HIV. Lopinavir is a protease inhibitor drug used in combination with ritonavir in therapy and prevention of HIV infection. Both drugs display polymorphism, which may lead to severe commercial implications for pharmaceutical manufacturing [1]. One way to overcome such problems is the development of amorphous formulations like Kaletra commercial medicine. In this work, we use a ball-mill system and an agate mortar and pestle to obtain individual amorphous ritonavir and lopinavir samples as well as their mixtures. First of all, we identify and characterize the crystal forms present in the raw samples of lopinavir and ritonavir, as well as quantify the mass concentrations of each crystal phase using X-ray powder diffraction and the Rietveld method. Ritonavir tends to recrystallize after some time. On the other hand, the mixture of an already amorphous lopinavir sample with ritonavir seems to facilitate its amorphization. The ball-mill processing of ritonavir and lopinavir together results in the production of unexpected crystalline forms of ritonavir. Pair distribution function (PDF) analysis shows the mixed samples reveal a higher $r$-dependence of ritonavir up to $2 \AA$ (first two peaks). At the same time, the lopinavir dependence tends to increase for a higher- $r$ signal.

[1] Bauer, J., Spanton, S., Henry, R., Quick, J., Dziki, W., Porter, W., Morris, J. (2001) Pharm. Res. 18, 859.

Keywords: Ritonavir; lopinavir; ball milling; pair distribution function; amorphous

The authors thank the financial support provided by The São Paulo Research Foundation - FAPESP (funding 2017/16499-5) and by the National Council for Scientific and Technological Development (funding 305601/2019-9). 